\title{
Relevamiento con drones; el caso Real de San Carlos
}

Drone mapping: case study: Real de San Carlos

\author{
> Arq. Marcelo Payssé Alvarez \\ Facultad de Arquitectura; \\ Universidad de la República, Uruguay \\ paysse@farq.edu.uy
}

\begin{abstract}
Cultural landscapes, although complex realities, give shape and meaning to the tangible and intangible components which form the foundations of human cultural background. The architecture of the city of Colonia del Sacramento fits within this context, and builds up a unique cultural landscape inserted in the logic of the historical heritage protection system. This concept, which implies wealth but also conflicts, demands a multidisciplinary approach grounded on a wide vision of this issue. Likewise, this comprehensive approach contributes to reverse and correct the lack of capacity and/or interest to save these examples of man-made landscape (prefabrication, mega-projects of the beginnings of the 20th Century, etc.), which are essential pieces of heritage conservation. Since 1943 the bullring is owned by the Municipality of Colonia. In the last decade the building was fenced to prevent breakdown risk. Still, illegal access occurs quite easily, increasing the risk and potential damage, in addition to the spoilage caused by more than one hundred years of inactivity and lack of maintenance. This paper proposes a method to survey and record the current status of the building, from photos taken by unmanned aerial vehicles (UAV, drones), allowing the registry without the need of direct access to the site. The survey will enable three types of results: series of mapped photographs, 3D models and an interactive platform for aerial view. The aim is to provide valuable and essential documentation for next stages of consolidation works, competitions and eventualy, new uses of the heritage building.
\end{abstract}

Reywords: registro de la forma; UAV; patrimonio

\section{Introducción}

La Plaza de Toros "Real de San Carlos" ubicada cerca de Colonia del Sacramento (Uruguay) es un edificio patrimonial de principios de siglo XX, y que se encuentra en condiciones muy comprometidas desde el punto de vista estructural.

Desde 1943 la Plaza de Toros es propiedad de la Intendencia de Colonia. En la última década el edificio fue cercado con tejido perimetral por riesgo de colapso.

Aún así, el acceso clandestino se produce con cierta facilidad, agravando la situación de riesgo y aumentando las posibilidades de deterioro directo, que se suman al causado por más de cien años de inactividad y falta de mantenimiento.

El presente trabajo propone un método de relevamiento y registro de la situación actual del edificio, mediante fotografías obtenidas utilizando vehículos aéreos no tripulados (UAV, drones), contemplando así la posibilidad de su registro sin la necesidad del acceso directo al predio.

El relevamiento permitirá obtener tres tipos de resultados: series de fotografías mapeadas, modelo 3D y una plataforma navegable tipo aerial view.

De esta manera se ofrecerá documentación valiosa e imprescindible para las próximas etapas de trabajos de consolidación, concursos y posibles nuevos usos del edificio patrimonial.

\section{Antecedentes}

La Plaza de Toros Real de San Carlos es parte del complejo turístico de comienzos del siglo XX desarrollado por el empresario naviero
Nicolás Mihanovich. El Complejo toma su nombre del lugar histórico donde se construye: la zona del campamento sitiador del gobernador Pedro de Cevallos que en 1761 se instala en ese lugar estratégico, próximo al río en comunicación con Buenos Aires, de forma de poder controlar los movimientos de la ciudad amurallada dominada por los portugueses.

Por ser campos del Rey Carlos III de España se llamó a esas tierras Real de San Carlos.

La Plaza de Toros RSC es un edificio que por su estilo y dimensiones, fue destacada en su época como una de las mejores, como las de primera categoría de España. Es la única de su tipo que en nuestro país y en la región se conserva en pie.

A finales del siglo XIX existían en nuestro país plazas en Montevideo, Salto, Paysandú, Colonia, Mercedes, etc. El 9 de enero de 1910 se inauguran, aún sin terminar, las obras de la Plaza de Toros RSC, jornada para la cual concurrieron cerca de 6.000 personas en 10 vapores desde Buenos Aires y en un tren expreso proveniente de Rosario, Sauce y Colonia Suiza.

Se realizaron veintitrés temporadas entre Abril de 1910 y Febrero de 1912, fecha de la clausura definitiva.

\section{Propuesta}

La primera manifestación de la propuesta recurrió a la utilización de las nuevas tecnologías de la información y la comunicación a los efectos de sensibilizar sobre el carácter y estado del edificio, para luego proponer aplicaciones interactivas que complementaran los recursos tradicionales. 


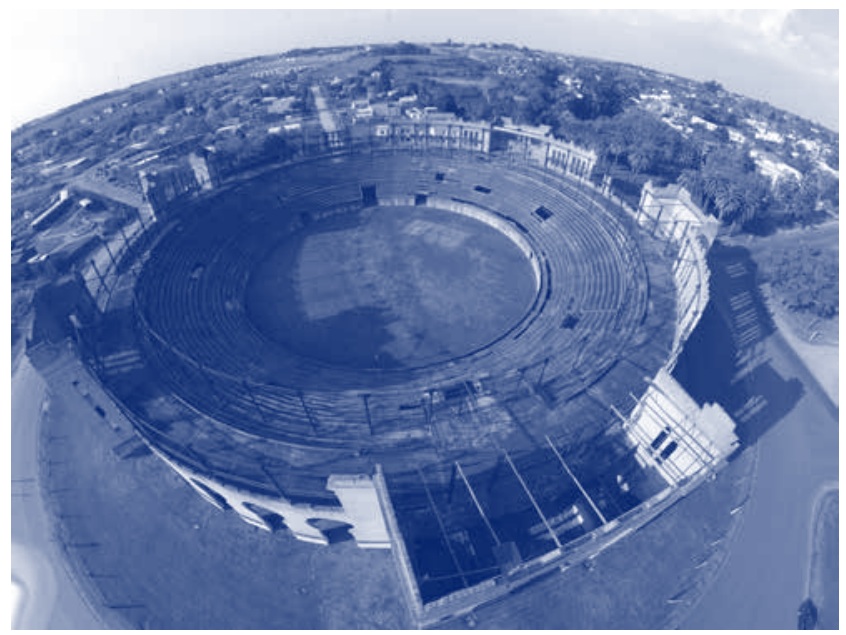

Figura 1: Vista desde el aire de la Plaza de Toros "Real de San Carlos.

El plan de acción supone el relevamiento, modelado y generación de aplicaciones interactivas, para la puesta en valor de este edificio histórico de Colonia del Sacramento y la posterior elaboración de maquetas tanto digitales como físicas.

Con tecnología de última generación instalada en el Laboratorio de Visualización Digital Avanzada (vidiaLab) de la Universidad de la República, se procura concretar todas las etapas del relevamiento necesarias para la concreción de los objetivos estipulados.

Dicho relevamiento en su fase completa, implica un registro de la realidad en su estado actual a los efectos de alimentar la información para el modelado digital 3D, y también la emisión de recaudos para las futuras intervenciones en el lugar.

Las aplicaciones interactivas permitirán la incorporación de información digital en el sitio y además abrirán la puerta a la oportuna exposición del trabajo realizado, en el Centro de Visitantes BIT de la ciudad de Colonia utilizando las nuevas tecnologías de la información y la comunicación.

Es sabido que difusión, conocimiento y valoración son factores inherentes a la gestión, y este proyecto se orienta hacia esos puntos. En ese sentido algunos de los objetivos serían los siguientes:

- Poner en valor el edificio patrimonial a través de aplicaciones interactivas de uso general.

- Integrar el edificio y su entorno al circuito turístico y cultural de la Ciudad de Colonia del Sacramento. Si bien esta integración existe en parte, se aspira a optimizarla.

- Registrar con detalle la situación existente como punto de partida de futuras intervenciones en el lugar.

- Dar un paso importante dentro de un proceso integral de gestión, capaz de incluir la recuperación y propuesta de nuevos usos del edificio.

- Coordinar diversas iniciativas que complementen la información sobre la ciudad de Colonia del Sacramento.

El plan de acción dispuesto para llevar adelante esta propuesta se encuentra en funcionamiento desde hace meses y desde octubre 2013 se realizan gestiones para difundir la iniciativa y así obtener los apoyos institucionales y económicos necesarios para lograr un adecuado cumplimiento de los objetivos.

Asimismo, como avance en sintonía con los fines del proyecto, se incluyó durante las actividades previstas para el Día del Patrimonio 2012, la presentación en el Centro Politécnico del Cono Sur de la maqueta de la Plaza Real de San Carlos realizada mediante fabricación digital en el Laboratorio de Fabricación Digital Montevideo (labFabMVD) de la Facultad de Arquitectura. Durante esa instancia, se presentaron en sociedad las ideas fuerza de la propuesta.

Esto supone distintas líneas de actuación que involucran disciplinas transversales al servicio de la difusión del proyecto y del acercamiento del mismo al contexto social. Entre ellas, corresponde especificar las relacionadas con las TICs, como punto de partida general:

- Relevamiento digital a través de fotografías y modelado $3 \mathrm{D}$ a partir de las mismas.

- Digitalización a partir de los planos y demás recaudos gráficos y escritos.

- Fabricación digital del proyecto, generando modelos físicos de distinta escala y detalle, aptos para presentaciones y estudios técnicos.

- Creación de aplicaciones de realidad aumentada, para smartphones y tabletas. De este modo, se crearán herramientas de comprensión del edificio al servicio del usuario, que incluyan geo-localizaciones, proyecciones, etc.

Centralización de toda la información del proyecto en un portal web actualmente en funcionamiento, llamado "Real de San Carlos: hacia una gestión integral".

\section{Metodología y materiales}

El edificio a relevar es de forma aproximadamente cilíndrica, con elementos que sobresalen, y con ritmos y módulos que se repiten radialmente. Estas características permiten reconocer elementos significativos que pueden estudiarse por separado.

Se realizarán series de fotografías que luego puedan ser identificadas mediante un código que refiera a la zona registrada y a la secuencia temporal en la que se encuentra.

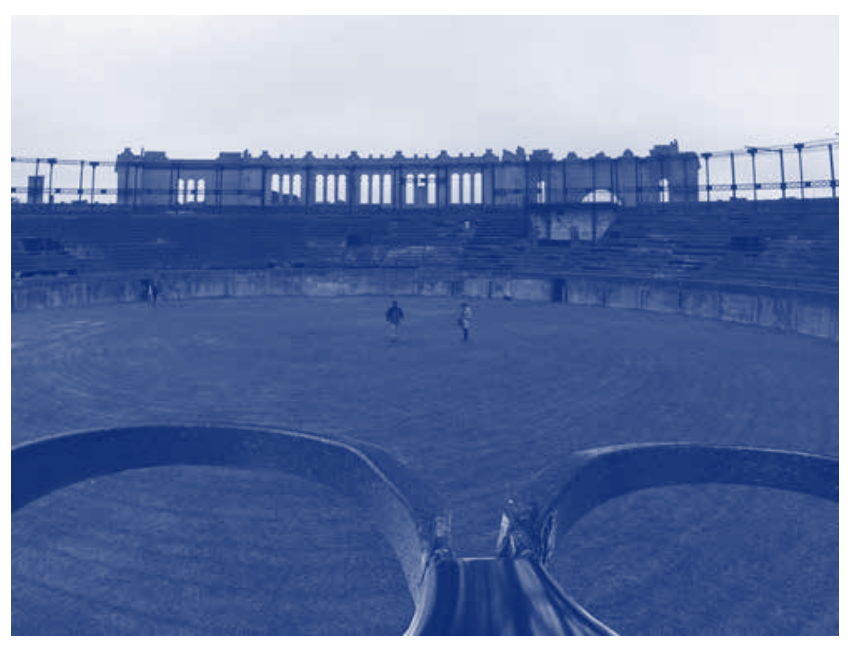

Figura 2: Relevamiento con drones desde ruedo interior. 


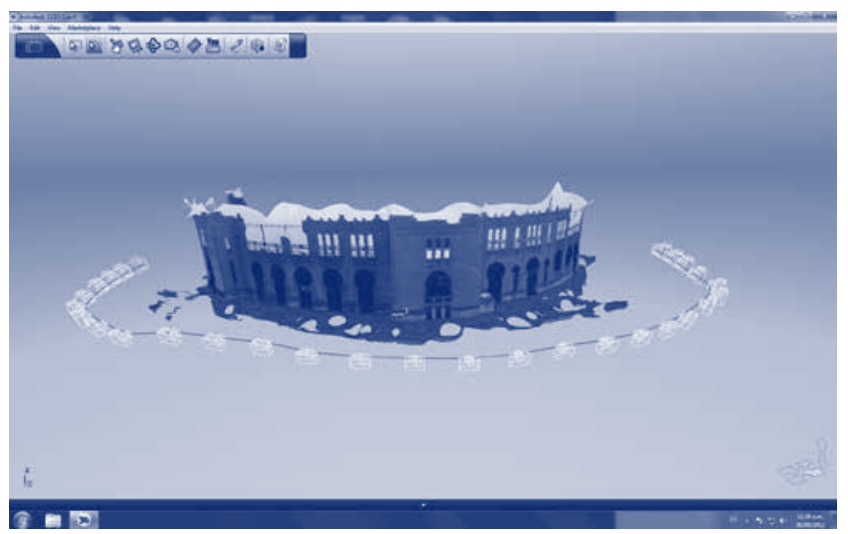

Figura 3: Primeros resultados del modelo de malla (123D Catch) obtenido por el vuelo perimetral.

Las fotografías serán obtenidas a partir de vuelos programados a cuatro alturas diferentes: a nivel de peatón (2mts), a nivel de mitad de muro exterior ( $8 \mathrm{mts})$, a nivel de coronamiento (16mts) y sobre el edificio (25mts). Estas series se repetirán tanto en el exterior como en el interior de la plaza, y por dentro del edificio.

Para cada serie se obtendrán unas 100 imágenes en promedio, lo que supone un número aproximado a las 1200/1400 fotografías.

En realizarán dos tipos de tomas exteriores: desde el espacio exterior (calle) y desde el interior (ruedo). En cuanto al espacio bajo gradas, se utilizarán ayudas como ser extensores de apoyo de cámaras par acceder a lugares complicados, a los que no puedan llegar los dispositivos aéreos.

Para el registro desde el aire se contará con drones tipo DJI Phantom 2, equipados con estabilizador Zenmuse H3 2D, cámaras GoPro Hero 3+ y sistema FPV.

Las tomas deberán ser editadas luego para compensar las deformaciones propias del gran angular y correcciones necesarias debidas a contraste y color.

Las series serán procesadas con 123D Catch, VisualSfM y CMPMVS para obtener los modelos de malla, y con Tourweaver para la generación de plataformas navegables.

\section{Resultados}

La documentación geométrica y fotográfica obtenida será un material de consulta para futuras y urgentes intervenciones sobre el edificio que permitan su consolidación y posterior puesta en valor y asignación de nuevos usos.

Este trabajo se propone el triple objetivo de sensibilizar sobre determinadas situaciones que requieren una urgente actuación, impulsar la recuperación patrimonial mediante diferentes iniciativas interdisciplinares en el ámbito de las TICs e implementar la puesta en valor y recuperación de la Plaza Real de San Carlos como paisaje antropizado y como elemento inmerso dentro de su propia dinámica social.

Asimismo, con el material producido, se realizarán maquetas a escala, tanto conceptuales como de detalle, a los efectos de complementar la información gráfica y fotográfica con la que se cuenta en este momento.

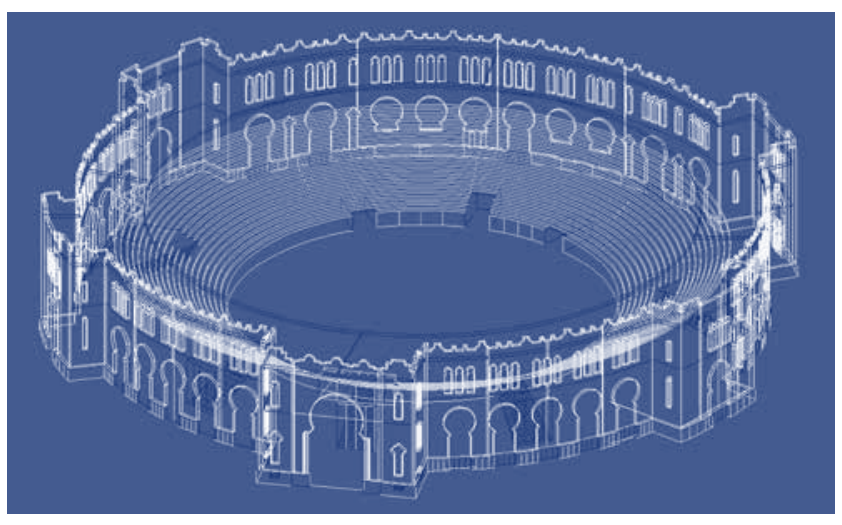

Figura 4: Modelo 3D con la geometría cooregida.

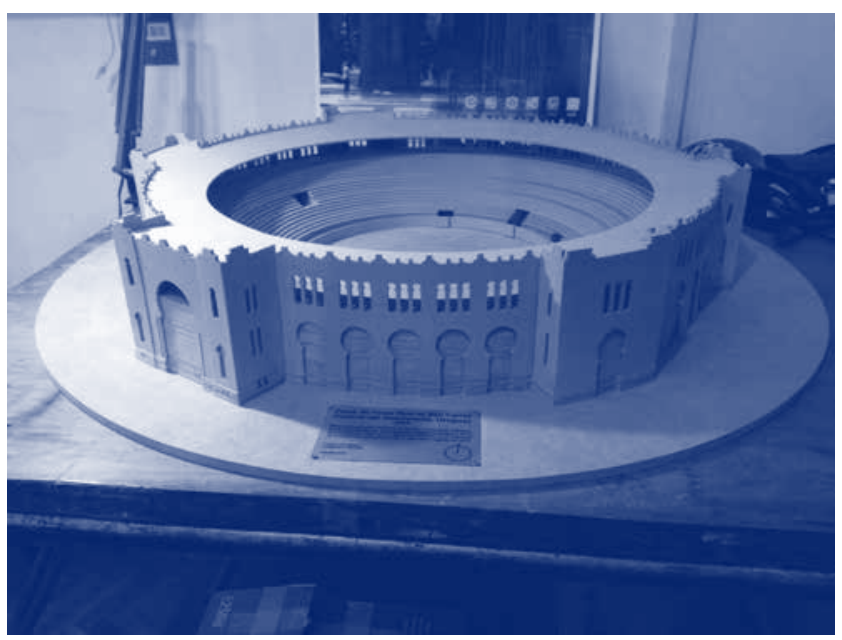

Figura 5: Maqueta del conjunto, realizada con fabricación digital.

Como resultado de esta iniciativa, se pretende realizar un aporte teórico-práctico a la recuperación patrimonial del proyecto, y al mismo tiempo, su revalorización en tanto que paisaje cultural.

Asimismo podrá integrarse al sistema de recorridos turísticos que se ofrecen como material digital en el Centro de Bienvenida, Interpretación y Turismo de Colonia (BIT Colonia).

En el campo académico significará una oportunidad para condensar los conocimientos generados en estos últimos dos años de registro de la forma con drones. Las actividades y metodologías utilizadas pasarán a integrar un protocolo de procedimientos y nuevas prácticas, a ser tenidas en cuenta para futuros relevamientos patrimoniales.

\section{Debate}

La Facultad de Arquitectura de la Universidad de la República no ha procesado debidamente la incorporación a su currícula de visiones y herramientas relacionadas con las nuevas tecnologías. Los últimos planes de estudio han ignorado llamativamente la necesidad de actualizar el discurso y la práctica del diseño en función de nuevos paradigmas aportados por las TICs.

Con la propuesta que se presenta, se intenta incorporar al debate académico una actividad de investigación, que pueda ser 
un antecedente válido para integrar definitivamente los medios digitales en las carreras de diseño.

Corresponde destacar que las líneas de acción promovidas por el proyecto Real de San Carlos, aun mientras esperan la necesaria e impostergable financiación, sirven de impulso a un cambio esencial de mentalidad y de visión, cambio en el que deberán involucrarse actores políticos, sociales y técnicos en beneficio de la salvaguarda del patrimonio cultural nacional, y por supuesto, del paisaje cultural universal.

\section{Referencias bibliográficas}

Payssé, M; Odriozola Guillot, M.A; García Amen, F.; Assandri, J.J.; Medina, M.; Paisaje, Patrimonio, Proyecto, Desarrollo Local;
Paisajes Culturales en Uruguay. Publicación CSIC. 2014.

Payssé, M; Montemuiño, E.; La tecnológica al servicio del patrimonio. Revista HABITAT. Reciclaje y restauración, v.: 64, p.: $114-118,2011$.

Remondino, F.; Campana, S.; 3D Recording and Modelling in Archaeology and Cultural Heritage; Theory and best practices. BAR International Series 2598. Archaeopress, Publishers of British Archaeological Reports. England, 2014.

Rekittke, Joerg; Ninsalam, Yazid; Paar, Philip.; No Fear of Ridicule - deploying plaything technology for credible representationes of urban landscapes. Págs. 28 a 46. IJAC Issue I, Volume 12; March 2014. 